\title{
Judicious Power Loading for a Cognitive Relay Scenario
}

\author{
Taskeen Nadkar, Vinay Thumar, U.B. Desai, S.N. Merchant \\ Indian Institute of Technology Bombay, 400076, India \\ Email:\{taskeenn,vinay_thumar,ubdesai,merchant\}@ee.iitb.ac.in Tel:+91-22-25764478
}

\begin{abstract}
This paper introduces a power loading strategy for relays to be used in a cognitive scenario. A cognitive relay node allows unlicensed (secondary) users to concurrently operate with licensed (primary) users, thereby improving spectral efficiency. The dynamic spectrum access in an OFDM-based cognitive relay network may lead to unwanted interference to the primary user from the side lobes of the secondary users. Two judicious algorithms are formulated by means of which the cognitive relay meets the twofold challenge of achieving optimum system throughput while ensuring minimum interference to the primary. The simulation results demonstrate that the suggested algorithms exhibit enhanced channel capacity for the secondary users as compared to traditional power allocation methods.
\end{abstract}

Keywords: Cognitive Radio, Relays, OFDM, Interference, Power loading

\section{INTRODUCTION}

In recent times, with the proliferation of wireless services, the legacy command and control spectrum regulation has created a discrepancy between spectrum allocation and its use. Cognitive Radio (CR) has been proposed as a mean to bridge the gap by flexible usage of the spectrum. It can be defined as a transceiver that can combine its awareness of the environment with knowledge of its user's needs, and adapt its parameters intelligently to achieve highly reliable communication [1][2]. Spectral efficiency can be improved by giving opportunistic access of licensed frequency bands meant for primary users (PUs) to a group of potential users, referred to as secondary users (SUs). Most of the current research in CR encompasses the following broad areas: spectrum sensing (to detect spectrum holes), transmit power control (to maximize the data rate of the secondary users), dynamic spectrum management (to distribute the unused spectrum fairly among the secondary users) [2]. Cooperative networks of cognitive nodes are proposed for improved efficiency and reliability of communications [3][4].

Orthogonal frequency division multiplexing (OFDM) is a mature modulation technique due to its ability to handle fading and inter-symbol interference. It presents a promising solution to enable flexible spectrum access in CR networks by dynamically nulling those subcarriers where the PU claims its spectrum [5][6]. However, it is observed that the OFDM system of SUs causes interference to the PUs limiting their performance. The Federal Communications Commission's (FCC) Spectrum Policy Task Force has recommended a metric called the interference temperature, which is intended to quantify and

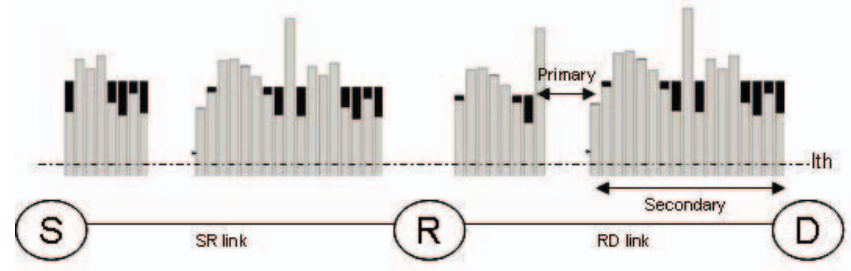

Fig. 1. OFDM based source-destination pair using a single cognitive relay

manage the sources of interference in a radio environment [2]. Any transmission in the frequency band of interest (the PU band in the case of CR networks) is considered to be harmful if it increases the noise floor above the interference temperature limit. In an OFDM-based SU system, the amount of interference to the primary band depends on the power allocation in the secondary's subcarriers, as well as the spectral distance between the subcarrier and the PU's band. The issue of interference mitigation in the primary band is receiving increasing attention in recent literature. Power loading algorithms for CR have been proposed and implemented in [7][8]. They are directed at maximizing the channel capacity for the SUs while minimizing the interference to the PUs.

Relays have gained popularity in recent times due to their ability to extend coverage of wireless networks. Relay nodes use either amplify-and-forward (AF) or decode-and-forward (DF) strategies to transmit data. DF relays have the advantage that the transmission at the source-relay (SR) and the relaydestination (RD) links (Figure 1) can be optimized separately. Moreover, the signal is regenerated at the relay, so that the noise is not amplified. Various aspects of relays for OFDM systems have been addressed in contemporary publications. Power allocation strategies for DF relays are proposed in [9][10].

The incorporation of $\mathrm{CR}$ technology in a relay creates a cognitive relay node ( $\mathrm{CogRel})$. A CogRel successfully achieves the functionality of a relay by improving the capacity between a given source-destination pair and increasing the coverage of the network. By virtue of its CR feature, it concurrently allows primary and secondary communication ensuring least interference to the PU and optimum throughput of the SU.

The preliminary model and functionality of a cognitive relay has been discussed in [11][12]. In [13], the authors have suggested a Cooperative Relay Scheme to increase the 
SINR at secondary receivers and defined a game to study the competition for shared spectrum among SUs. In [15] the mutual interference channel for cognitive relays has been investigated. Improved secondary performance by appropriate power allocation and beamforming in a heterogeneous cognitive relay system is discussed in [14].

In this paper we have simulated an OFDM-based CogRel for a single source-destination pair, which pose as secondary users. The CogRel has the ability to sense the appearance of the PU of the spectrum and null the secondary's subcarriers accordingly on both the SR and RD links. Unique power allocation algorithms are formulated and implemented. The first algorithm follows a convex optimization approach to maximize the link channel capacity under a constrained primary interference. The second algorithm is an innovation of traditional water-filling. It iteratively distributes power among the subcarriers based on the channel conditions and simultaneously maintains the interference to the primary below a specified threshold. Both the algorithms enhance the communication individually on the SR and RD links. To further improve the end-to-end system throughput, a subcarrier permutation is implemented. With the use of sound mathematical expressions, corroborated by numerical results of simulation, a CogRel is demonstrated to be a novel extension to the existing work in CR technology and wireless relays. To the best of our knowledge, the power allocation strategy proposed for cognitive relays is unprecedented in literature.

To detail the proposed scheme, the paper has been organized as follows: Section II describes the system model and radio environment. Section III methodically explains the CogRel implementation and outlines the power allocation algorithms. Section IV presents comparative numerical results and Section $\mathrm{V}$ concludes the paper.

\section{System Model AND COMmunication Scenario}

An OFDM-based secondary user system is considered with $\mathrm{N}$ subcarriers. It consists of a source-destination pair assisted by a single relay $(\operatorname{CogRel})$. The relay uses DF strategy. It is assumed that the distance between the source and destination is too large for them to communicate directly. The channel state information (CSI) is available to the source, relay and destination. Different channels experience independent fading. There exists a PU in the radio range of the relay. When the PU claims its frequency band, the CogRel detects it and conveys the information to both the source and destination. The corresponding subcarriers of the secondary are then nulled. If the primary requires bandwidth equivalent to $\mathrm{N}_{p}$ subcarriers, then there are $\mathrm{N}_{a}=\mathrm{N}-\mathrm{N}_{p}$ active subcarriers.

If the data on the $\mathrm{i}^{\text {th }}$ subcarrier on the SR link is relayed on the $\mathrm{j}^{\text {th }}$ subcarrier of the RD link, the end-to-end channel capacity of the subcarrier pair $\mathbf{R}_{i j}$ is given by eqn. 1 [9]. $\mathbf{R}_{s i}$ and $\mathbf{R}_{r j}$ are the channel capacities on the $\mathrm{SR}$ and $\mathrm{RD}$ links respectively (interference from the primary to the secondary is assumed to be negligible).

$$
\begin{aligned}
R_{i j} & =\min \left(R_{s i}, R_{r j}\right) \\
R_{s i} & =\frac{B}{2 N} \log _{2}\left(1+\frac{P_{s i} h_{s i}}{\sigma^{2}}\right) \\
R_{r j} & =\frac{B}{2 N} \log _{2}\left(1+\frac{P_{r j} h_{r j}}{\sigma^{2}}\right)
\end{aligned}
$$

where $\mathrm{B}$ is the total bandwidth of OFDM , $\sigma^{2}$ is the AWGN variance, $\mathrm{P}_{s i}$ and $\mathrm{P}_{r j}$ are the powers distributed on the individual subcarriers on the SR and RD links respectively, $\mathrm{h}_{s i}$ and $\mathrm{h}_{r j}$ are the corresponding channel power gains.

Due to the coexistence of the primary and secondary, the side lobes of the OFDM-based SU interfere with the primary's signal. The interference power introduced to the PU band is the integration of its power spectral density. Interference induced to the primary by the $\mathrm{i}^{\text {th }}$ subcarrier of the secondary OFDM system on the SR link is given by [7]:

$$
I_{s i}=P_{s i} T_{s} \int_{\text {PUband }}\left(\frac{\sin \pi f T_{s}}{\pi f T_{s}}\right)^{2} d f
$$

where $\mathrm{P}_{s i}$ is the power emitted by the $\mathrm{i}^{\text {th }}$ subcarrier and $\mathrm{T}_{s}$ is the symbol duration. Similarly, interference induced to the primary by the $\mathrm{j}^{\text {th }}$ subcarrier of the secondary OFDM system on the RD link is given by:

$$
I_{r j}=P_{r j} T_{s} \int_{\text {PUband }}\left(\frac{\sin \pi f T_{s}}{\pi f T_{s}}\right)^{2} d f
$$

where $\mathrm{P}_{r j}$ is the power emitted by the $\mathrm{j}^{\text {th }}$ subcarrier

The goal is to distribute the power effectively on the SR and RD links to ensure superior system throughput. It is a well established fact that most favourable power allocation is waterfilling. However, a traditional waterfilling will not work in a radio environment where the primary and secondary users co-exist. It is critical to maintain the interference to the primary band below a specified threshold. Furthermore, to achieve enhanced capacity on the source-destination pair, subcarriers need to be matched in accordance with the subcarrier powerchannel gain product i.e. $\mathrm{P}_{i}-\mathrm{h}_{i}$ (eqns. 2,3). This is because the fading gains of different channels are mutually independent; the subcarriers which experience deep fading over the SR link may not experience deep fading on the RD link. As such, the channel capacity of the system (eqn. 1) will be limited by the worst subcarrier. Hence, the data on the subcarrier on the SR link is reallocated to some other subcarrier on the RD link based on the power- channel gain product [9]. The task is performed at the CogRel and results in optimum system channel capacity.

\section{Proposed Scheme}

A novel strategy is suggested to relay SU's data in a dynamically changing radio environment. The implemented $\mathrm{CogRel}$ has the intelligence to monitor and adapt to the changes. Two unique power allocation schemes are formulated, which meet the challenge of enhancing the channel capacity while maintaining interference to the primary under the specified limit. 


\section{A. Scheme A : Primary Interference Iterative Waterfilling}

An iterative waterfilling algorithm is implemented to allocate the power budget $\mathrm{P}_{s}$ on the SR link based on channel conditions while ensuring that the interference to the primary is below a specified threshold $\left(\mathrm{I}_{t h}\right)$. The algorithm operates as follows: (i) Since the SU band closest to the PU band causes maximum interference to it, power distribution starts from the channel closest to the PU band in accordance with the inverse channel gains. (ii) After every allocation, the interference power in the primary band $\left(I_{s}=\sum I_{s i}\right)$ is computed and compared with the set threshold $\left(\mathrm{I}_{t h}\right)$. If it exceeds the threshold, the power in the $\mathrm{i}^{\text {th }}$ subcarrier is reduced proportionally and the differential power is redistributed in the remaining subcarriers. (iii) The process is repeated iteratively for subcarriers while progressing away from the primary band, till the interference is below the threshold.

A similar procedure is implemented on the RD link with a power budget $\mathrm{P}_{r}$.

\section{B. Scheme B : Constrained Optimization}

The disadvantage of Scheme $\mathrm{A}$ is that waterfilling has to be implemented several times till convergence occurs. Constrained optimization is a sophisticated approach to the situation. The problem is formulated on the SR link as follows:

$$
C_{s r}=\max \sum_{i=1}^{N_{a}} R_{s i}\left(h_{s i}, P_{s i}\right)
$$

such that

$$
\sum_{i=1}^{N_{a}} I_{s i}\left(d_{s i}, P_{s i}\right) \leq I_{t h}
$$

and

$$
P_{s i} \geq 0
$$

Similarly, on the RD link, we have

$$
C_{r d}=\max \sum_{j=1}^{N_{a}} R_{r j}\left(h_{r j}, P_{r j}\right)
$$

such that

$$
\sum_{j=1}^{N_{a}} I_{r j}\left(d_{r j}, P_{r j}\right) \leq I_{t h}
$$

and

$$
P_{r j} \geq 0
$$

where $\mathrm{d}_{s i}$ represents the spectral distance between the $\mathrm{i}^{\text {th }}$ subcarrier on the SR link and the corresponding PU band, and $\mathrm{d}_{r j}$ represents the distance between the $\mathrm{j}^{\text {th }}$ subcarrier on the RD link and the PU band.

With $\lambda_{1}$ and $\lambda_{2}$ as the Lagrange constraints, the Lagrange formulation for the SR and RD links is given respectively by

$$
L_{s r}=\sum_{i=1}^{N_{a}} R_{s i}\left(h_{s i}, P_{s i}\right)-\lambda_{1}\left(\sum_{i=1}^{N_{a}} I_{s i}\left(d_{s i}, P_{s i}\right)-I_{t h}\right)
$$

$$
L_{r j}=\sum_{j=1}^{N_{a}} R_{r j}\left(h_{r j}, P_{r j}\right)-\lambda_{2}\left(\sum_{j=1}^{N_{a}} I_{r j}\left(d_{r j}, P_{r j}\right)-I_{t h}\right)
$$

The solution to the optimization problem yields the optimum power per subcarrier on the SR and RD links respectively as

$$
P_{\text {siopt }}=\max \left(\frac{1}{\lambda_{1} * \frac{\partial I_{s i}}{\partial P_{s i}}}-\frac{\sigma^{2}}{h_{s i}}, 0\right)
$$

and

$$
P_{\text {rjopt }}=\max \left(\frac{1}{\lambda_{2} * \frac{\partial I_{r j}}{\partial P_{r j}}}-\frac{\sigma^{2}}{h_{r j}}, 0\right)
$$

The Lagrange constraints are calculated as

$$
\lambda_{1}=\frac{N_{a}}{I_{t h}+\sum_{i=1}^{N_{a}} \frac{\sigma^{2} * \frac{\partial I_{s i}}{\partial P_{s i}}}{h_{s i}}}
$$

and

$$
\lambda_{2}=\frac{N_{a}}{I_{t h}+\sum_{j=1}^{N_{a}} \frac{\sigma^{2} * \frac{\partial I_{r j}}{\partial P_{r j}}}{h_{r j}}}
$$

\section{Relaying Strategy}

The communication from source to destination is completed in three phases:

1) Sensing and information sharing: The secondary communication is already in progress. The CogRel is sensing the radio environment for appearance of the primary. Once detected, the information is conveyed to the source and destination. The devices adapt to the new radio environment and subcarriers are nulled to accommodate the PU.

2) Source-to-relay transmission: Communication on the SR link occurs with the subcarrier power levels computed using either Scheme A or Scheme B.

3) Relay-to-destination transmission: The data received by the $\operatorname{CogRel}$ is decoded. Similar power allocation is implemented on the RD link. To achieve higher endto-end throughput, before the data is relayed on the RD link subcarriers are arranged in order of their powerchannel gain product and matched accordingly. The communication to the destination is now complete.

\section{Simulation RESUlTS}

A 64 subcarrier OFDM secondary system is considered. The PU requires 8 subcarriers to be nulled. Each subcarrier undergoes Rayleigh fading. The average channel power gains on the $\mathrm{SR}$ and RD links are assumed to be 1 , i.e. $\mathrm{E}\left(\mathrm{h}_{s i}\right)=\mathrm{E}\left(\mathrm{h}_{r i}\right)=1$.

The proposed power allocation algorithms are implemented at the source and relay. Subcarriers on the SR and RD links are paired for increased throughput. The source-destination channel capacity is computed in accordance with eqns. 1-3. The following schemes are compared (i) Optimized power allocation with subcarrier matching - The power allocation is based on Lagrange's optimization and subcarriers on the SR link are matched with those on the RD link in accordance 


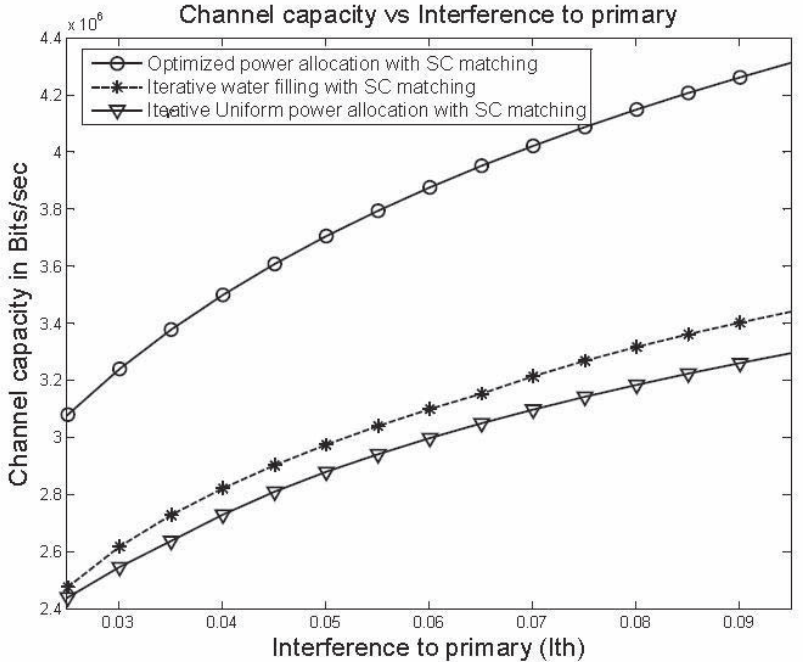

Fig. 2. Secondary user channel capacity

with the power-channel gain product.(ii) Iterative waterfilling with subcarrier matching- The power is distributed with the proposed iterative waterfilling algorithm and subcarriers are matched. (iii) Uniform power allocation with subcarrier matching - The power is distributed uniformly among the subcarriers irrespective of the channel conditions, iteratively while maintaining the primary interference below the threshold. Also subcarriers are matched.

It is observed that the optimized power allocation scheme with subcarrier matching gives best results, which is followed by iterative waterfilling. Iterative uniform power distribution exhibits the least channel capacity of the three methods compared, since it does not exploit channel conditions. Figure 2 shows channel capacity vs. primary interference threshold $\left(\mathrm{I}_{t h}\right)$ for various schemes.

\section{CONCLUSION}

Judicious power allocation algorithms have been formulated to be used in an OFDM-based cognitive relay - CogRel. The proposed algorithms: constrained optimization and primary interference iterative waterfilling are implemented on a single hop network. It is validated by simulation results that the suggested power allocation schemes not only maximize the end-to-end throughput of the secondary users, but concurrently mitigate the interference to the primary user band. The suggested CogRel strategy will enable spectrally efficient multihop networks, in which licensed and unlicensed users can coexist with superior system performance for both.

\section{ACKNOWLEDGEMENT}

This work was supported by Microsoft Corporation and Microsoft Research India under the Microsoft Research India PhD Fellowship Award 2009.

\section{REFERENCES}

[1] J. Mitola, "Cognitive Radio for Flexible Mobile Multimedia Communications," in IEEE International Workshop on Mobile Multimedia Communications, MoMuC '99, pp. 3-10, 1999.

[2] S. Haykin, "Cognitive Radio: Brain-Empowered Wireless Communications," IEEE Trans. Selected Areas in Communications, vol. 23, pp. 201220, Feb. 2005

[3] S.Mishra, A.Sahai,R. Brodersen, "Cooperative Sensing among Cognitive Radios", in Proc. of IEEE Int. Conf. on Communications, ICC '06, vol. 4, pp. 1658-1663, Jun. 2006

[4] W. Wang, L. Zhang, "On the Distributed Cooperative Spectrum Sensing for Cognitive Radio", International Symposium on Communications and Information Technologies, ISCIT '07, pp. 1496-1501, Oct. 2007

[5] R. Rajbanshi, A. M. Wyglinski, and G. J. Minden, "An efficient implementation of NC-OFDM transceivers for cognitive radios," in Proc. of 1 st 180 Int. Conf. on Cognitive Radio Oriented Wireless Networks and Commun., June 2006.

[6] Ekram Hossain, Vijay K. Bhargava, "Cognitive Wireless Communication Networks", Springer, 2007

[7] Bansal, G.; Hossain, M.J.; Bhargava, V.K., "Adaptive Power Loading for OFDM-Based Cognitive Radio Systems," in Proc. of IEEE Int. Conf. on Communications, vol., no., pp.5137-5142, 24-28 June 2007

[8] Hasan, Z.; Hossain, E.; Despins, C.; Bhargava, V.K., "Power Allocation for Cognitive Radios Based on Primary User Activity in an OFDM System," in Proc. of IEEE Global Telecommunications Conference, IEEE GLOBECOM 2008. , vol., no., pp.1-6, Nov. 30 2008-Dec. 4 2008

[9] Wang, W., Yan, S., and Yang, S. "Optimally joint subcarrier matching and power allocation in OFDM multihop system," EURASIP J. Adv. Signal Process 2008

[10] Wenyi Wang; Shuyuan Yang; Li Gao, "Comparison of Schemes for Joint Subcarrier Matching and Power Allocation in OFDM Decode-andForward Relay System," in Proc. of IEEE Int. Conf. on Communications, vol., no., pp.4983-4987, 19-23 May 2008

[11] Mao Xiaomao; Qiu peiliang, "Cognitive Relay," Future Generation Communication and Networking (FGCN 2007), vol.2, no., pp.264-269, 6-8 Dec. 2007

[12] Chunyi Song; Shiba, H.; Shimamoto, S., "A cognitive relay approach for multi-hop wireless ad hoc communication," in Proc. of Third Int. Conf. on Communications and Networking in China, ChinaCom 2008, vol., no., pp.1230-1234, 25-27 Aug. 2008

[13] Xiaowen Gong; Wei Yuan; Wei Liu; Wenqing Cheng; Shu Wang, "A Cooperative Relay Scheme for Secondary Communication in Cognitive Radio Networks," in Proc. of IEEE Global Telecommunications Conference,IEEE GLOBECOM 2008., vol., no., pp.1-6, Nov. 30 2008-Dec. 4 2008

[14] Chunhua Sun; Ben Letaief, K., "User Cooperation in Heterogeneous Cognitive Radio Networks with Interference Reduction," in Proc. of IEEE Int. Conf. on Communications, vol., no., pp.3193-3197, 19-23 May 2008

[15] Mingkang Cheng; Jingli Mao; Lee Li, "Investigation of Mutual Interference Channel with Relay in Cognitive Transmission," in Proc. of 4th Int. Conf. on Wireless Communications, Networking and Mobile Computing, WiCOM '08. , pp.1-4, 12-14 Oct. 2008 